\title{
Growth Phenotype Screening of Schizosaccharomyces pombe using a Lensless Microscope
}

\author{
Lynsey A. Penwill ${ }^{\mathrm{a}}$, Gwendoline E. Batten ${ }^{\mathrm{a}}$, Stefania Castegnetti ${ }^{\mathrm{b}}$ and Andrew M. \\ Shaw $^{\mathrm{a} *}$ \\ ${ }^{a}$ Biosciences, College of Life and Environmental Sciences University of Exeter Stocker Road Exeter UK \\ Fax: 01392 723434; Tel: 01392723495 \\ b 1-UPMC Univ Paris 06, Laboratoire de Biologie du Développement de Villefranche-sur-mer (LBDV), \\ Observatoire OcéanoLOGIQUE, 06230 Villefranche-sur-mer, France. \\ 2-CNRS, Laboratoire de Biologie du Développement de Villefranche-sur-mer (LBDV), Observatoire \\ OcéanoLOGIQUE, 06230 Villefranche-sur-mer, France \\ *Corresponding Author: E-mail: Andrew.M.Shaw@exeter.ac.uk
}

The Lensless microscope has a large field of view and allows the capture of the diffraction pattern from a large number of cells simultaneously. A simple algorithm to measure intensity changes in the Airy Disc First Fringe (ADFF) has been derived to follow the growth characteristics of the single cell yeast Schizosaccharomyces pombe. The performance of the algorithm is calibrated using comparison between optical image and ADFF analysis of polystyrene microspheres with known dimensions and has an accuracy of 5\% over all lengths above the diffraction-limited measurements. We have observed the growth characteristics of $S$. pombe for $N=100$ cells to determine the growth phenotype distributions of length and width on arrival at the surface, lag phase adjustment to the new growth conditions, the length at birth, $L_{B}$, and cell cycle length, $t_{\text {cell }}$. The observed cell width distribution has a median width of $3.9( \pm 0.1) \mu \mathrm{m}$, as expected, but a non-normal distribution. Similarly, all growth parameters studied, $L(t=$ $0), L_{B}$ and cell cycle time are phenotypes with non-normal distributions but with medians consistent with the literature values.

'Lensless Microscope', 'Phenotype Screening', 'Rapid Detection', 'High-throughput cell screening' 


\section{Introduction}

A Lensless microscope is a diffractive imaging device that measures the scattered light intensity from a scattering object without any focussing optics (Isikman et al. 2011; Tseng et al. 2010). The diffraction pattern or Airy Disc contains all of the information required to re-construct digitally the microscope image (Schnars and Jueptner 2005). The first Lensless microscope study imaged the growth behaviour of the nematode worm Caenorhabditis elegans (Lange et al. 2005) over time. These studies have been extended to observe large numbers of cells and variations within their populations in a rapid, simple and cost effective manner (Ozcan and Demirci 2008). Like all optical imaging systems, the resolution of the Lensless microscope is limited by the diffraction limit. The diffraction limit is calculated as $\lambda / 2 \times \mathrm{NA}$ for systems illuminated by a single wavelength, $\lambda$, NA is the numerical aperture of the imaging lens (2009). The NA for Lensless microscope is 1 and the diffraction limit as $0.3 \mu \mathrm{m}$, for a white light source with peak wavelength of $647 \mathrm{~nm}$.

The advantages of the Lensless microscope have been detailed elsewhere (Seo et al. 2008) but two principal advantages are the cost of the optics and the significantly larger field of view. Imaging biological samples such as cells usually requires a $40 \times$ objective lens which typically has a field of view of $165 \times 220 \mu \mathrm{m}\left(0.036 \mathrm{~mm}^{2}\right), 10^{3}$ times smaller than a typical Lensless microscope field of view of $5.2 \times 7.2 \mathrm{~mm}\left(38 \mathrm{~mm}^{2}\right)$. The large field of view allows the simultaneous measurement of large number of cells or colonies and a simple imaging analysis technique has potential to make this a point-of-care rapid microbial identification (Peeling and Mabey 2010).

Lensless microscopes with full holographic image reconstruction (Bishara et al. 2010; Cui et al. 2008; Su et al. 2010; Xu et al. 2001) have been used to identify the different components of blood such as erythrocytes which are typically $7 \mu \mathrm{m}$ in diameter (Seo et al. 2010; Su et al. 2009). However, we seek to demonstrate that a simple image analysis without full image reconstruction is sufficient to interrogate the growth characteristics of single-cell deriving the changes in length and width of the scattering object.

The growth characteristics of cells growing in solution are almost impossible to determine with only population averages observed. However, growth on a surface allows individual cells and their colonies to be studied revealing some remarkable growth phenotypes such as persister cells (Lewis 2010), more generalised biofilm population dynamics (Hall-Stoodley et al. 2004; Sauer et al. 2002) and cancerous growth (Bendall and Nolan 2012). The individual phenotypes remain very difficult to study appearing in low numbers and losing their phenotype when transferred back to planktonic growth. The importance of characterising these phenomena for understanding infection and targeting tumours is significant and new screening technologies for rapid identification are required.

The potential for the Lensless microscope and simple Airy Disc image analysis to be a technology for screening growth phenotype is explored using the rod-shaped fission yeast, Schizosaccharomces pombe. There are several growth phenotypes that are promising candidates for screening. Transition from one medium to another induces an 
empirical lag period in the growth, $(\lambda)$, typically 2 hours, where the cell is metabolically active but not increasing in length. The yeast grows exclusively by tip elongation at cell ends (Snell and Nurse 1993) maintaining a highly controlled width of 3-4 $\mu \mathrm{m}$ (Mitchison and Nurse 1985). At birth fission yeast cells are 6-8 $\mu \mathrm{m}$ long and double in length before division by binary fission (Mitchison and Nurse 1985) at each cell cycle. After birth fission yeast cells are monopolar and grow only from the old end, which was already present in the mother cell before division. Between 0.3 and 0.4 of the cell cycle (where birth is 0 and septation is 1) (Martin and Chang 2005) the cells reach the Rate Changing Point (RCP) and the opposite, new end of the cell starts growing, increasing the overall rate of cell elongation. This transition from unipolar to bipolar growth is known as New End Take Off (NETO) (Snell and Nurse 1993). Cell growth ceases when cells enter mitosis ( 0.8 of the cell cycle) and resumes only after cytokinesis. The length of the cell cycle, the time from birth until cytokinesis, varies with temperature and growth conditions but in rich medium at $25^{\circ} \mathrm{C}$ is reported in the region of $3.1 \mathrm{hrs}$ (Fantes 1977; Mitchison and Nurse 1985).

In this paper, we aim to validate the potential of the Lensless microscope with simple Airy Disc analysis to identify the cell growth characteristics of $S$. pombe cells to determine growth phenotype distributions.

\section{Materials and Methods}

The Lensless microscope set up is displayed in Figure 1. An aperture of $100 \mu \mathrm{m}$ in diameter sits in front of a white light source, with a peak wavelength value of $627 \mathrm{~nm}$ (Thorlabs, MWWHF1, $7.0 \mathrm{~mW}$ ). The pinhole aperture creates a pseudo- point source, improving the coherency of the light waves without generating an interference pattern or restricting the field of view.

INSERT FIGURE 1 HERE

The light propagates over a distance, $d$, of $15 \mathrm{~cm}$ before reaching the flow cell where it will interact with the diffracting objects of interest, microspheres and S.pombe cells. The resulting Airy Disc pattern is collected on a Charge Coupled Device (CCD) camera (IM 3.2 , Jenoptik), set at a working distance, $z$, typically $1 \mathrm{~mm}$. The working distance is chosen to ensure the Airy Disc patterns cover $20 \times 20$ pixels on the camera but not allow the patterns from nearby cells to overlap. The flow cell is tightly clamped to the surface of the CCD camera to ensure the working distance is constant throughout the experiment. The flow cell is composed of two cover slips held either side of a silicone border with petroleum jelly. The flow cell has a constant depth of $\sim 1 \mathrm{~mm}$ and a total volume of $\sim 175 \mu \mathrm{L}$. The cell has entrance and exit flow ports to allow medium to enter and leave the flow cell: the ports remained closed in these experiments. The CCD chip is connected via IEEE 1394a FireWire to the operating computer. The images are collected in 8-bit vertical resolution and stored in TIFF format. The images are averaged and analysed using software written in Matlab $\odot$ (Matlab 1994-2013). Images are captured at a rate of 15 frames per second (24565 pixels). Airy Disc patterns are analysed using an algorithm to determine the intensity brightness between the centre and first fringe of the Airy Disc (Figure $1 \mathrm{C}$ ). The centre of the Airy Disc is specified by the user and is stable throughout the time course. A virtual mask is located over the centre and the first fringe and the 
intensity is then integrated as a function of angle. The major axis is defined as the axis of maximum intensity difference, $\Delta I$; the minor axis is defined perpendicular to the major axis.

The simple intensity measure along the major and minor axes leads to an ADFF method for monitoring the evolution of the Airy Disc over time. The software mask has automated corrections for 'jitter' and mask misalignment. The intensity difference along the major and minor axis is then monitored over time as the cells grow. A calibration curve was constructed using polystyrene microspheres (Sigma-Adrich Microparticles 80177, 72938, 95531, 59336, 87896, 88511, 72822 and 80304) with diameters in the range from 1-60 $\mu \mathrm{m}$. The intensity difference, $\Delta I$, along a fixed major axis was derived for 20 spheres of each diameter, collected during 4 replica experiments. For comparison, the diameter of the microspheres was also derived from images collected using a 40x microscope objective (Primo Star and AxioCam ERc5s, Carl Zeiss). The ADFF and optical microsphere diameters comparison is shown in Figure 1. Experimental $\Delta I$ values were converted to length using the calibration curve; the accuracy is diffraction limited at $\pm 0.15 \mu \mathrm{m}$.

\section{Cell Growth Methods}

S. pombe (WT 972 h-) was grown and handled using standard methods(Moreno et al. 1991). All experiments were performed in YE5S medium at $25^{\circ} \mathrm{C}$ (yeast extract with added $250 \mathrm{mg} / \mathrm{L}$ histidine, adenine, leucine, uridine and lysine).

A log-phase growth culture of $S$. pombe was diluted to OD $=0.01$ and $100 \mu \mathrm{L}$ of cell suspension was deposited in the flow cell, the lower surface of which was coated in lectin (Sigma-Aldrich, Lectin from Glycine max, L1395, 100 $\mathrm{g} / \mathrm{mL}$ ). After 2 minutes the excess solution was removed, leaving only the cells that had adhered to the lectin-coated surface. The chamber was filled with YE5S broth containing a 3-point calibration of

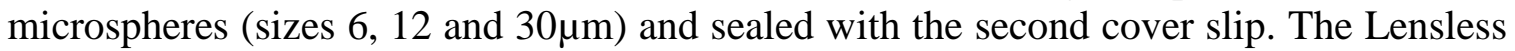
microscope was placed in an optically dark, temperature controlled chamber. The temperature of this chamber was maintained at $25^{\circ} \mathrm{C}( \pm 1)$, this remained stable over the time course of the experiment. The temperature of the growth cultures was maintained at $25^{\circ} \mathrm{C}$. The growth experiment was repeated 12 times, with each repeat containing between 6 and 12 cells.

\section{Statistical Analysis}

The Airy Discs for all 100 colonies were analysed during the growth period from which the growth parameters were derived. The (major axis) length of the cell at $t=0$, the (minor axis) width of the cell, Aspect Ratio (AR, the ratio of cell length and cell width), the birth length of the cell (half of the length at cytokinesis) $L_{B}$ and the cell cycle length, tcell are well known in the literature and were therefore derived as test growth parameters. All histograms are normalised to have an area of 1, derived from $N=100$ and tested for normality using the both Shapiro-Wilk (SW) test (Shapiro and Wilk 1965) and the Anderson-Darling (AD) test (Anderson and Darling 1952) as recommended by Henderson (Henderson 2006). The skewness value is obtained for each distribution, indicating whether the data are symmetrical (a value of 0 ), skewed to the left of the mean 
(a negative value) or skewed to the right of the mean (a positive value). The 95\% confidence limits were obtained by bootstrapping the median parameters to 1000 samples. All Box plots have whiskers (w) extending to the range of data. Outliers are defined as $\mathrm{q} 3+1.5(\mathrm{q} 3-\mathrm{q} 1)$ and smaller than $\mathrm{q} 1-\mathrm{w}(\mathrm{q} 3-\mathrm{q} 1)$ where $\mathrm{q} 3$ and $\mathrm{q} 1$ are the 75th and 25th quartile respectively are plotted individually. The Box plots are overlaid with distribution spread plots. All tests and analyses are implemented in Matlab.

\section{Results}

The calibration curve (Fig. 1 C) shows that the size of spheres calculated from the intensity measurements correlates strongly to the measurements determined optically, with an $\mathrm{R}^{2}$ value of 0.997 and a slope of 0.992 . Microscope images of 20 fission yeast cells we collected and compared with their corresponding Airy Disc (Fig. A. 1) to establish the Airy Disc characteristics of single cells and their dimension variability. Growth experiments were performed over a period of 9 days observing the growth of 100 single-cell colonies. A typical growth curve is shown in Figure 2 defining the growth parameters.

\section{INSERT FIGURE 2 HERE}

The distributions of the parameters in figure 2 have the potential to highlight cells with different phenotypes, for example there may be a percentage of cells with a significantly shorter lag period or longer length at cytokinesis. The normalised histograms of the width and length distributions derived from $N=100$ S. pombe cells are shown in Figure 3.

\section{INSERT FIGURE 3 HERE}

Neither of the cell length or width on arrival at the surface are normally distributed, (SW and $\mathrm{AD}-\mathrm{P}<0.001$ for both data sets). The cell length has a median value of $9.2(+0.6$ $0.9) \mu \mathrm{m}$ with a slight bias to shorter lengths (skewness coefficient 0.49 ). The distribution of cell widths at $\mathrm{t}=0$ is very narrow with a diffraction limited median of $3.9( \pm 0.15) \mu \mathrm{m}$ and a range of $1.8 \mu \mathrm{m}$ and a skewness coefficient of 0.19 , congruent with published data, Figure 3.

The diffraction pattern changes significantly at cytokinesis where the two cells have divided and begun to move apart at an angle to each other, Figure 2 (confirmed from microscope images and time-lapse videos). The time to cytokinesis (A) is the time taken for the cell to double in length and divide, the birth length, $L_{B}$ is defined as half of the length at A, corrected using the 1.11 correction factor for $S$. pombe dividing into two (Mitchison and Walker 1959). This factor corrects for the fact that as cells divide they develop hemispherical ends from cylindrical ones. The distribution for $L_{B}$ is shown in Figure 4.

\section{INSERT FIGURE 4 HERE}




\section{Discussion}

The size of spheres calculated from the intensity measurements correlates strongly to the measurements determined optically for identical spheres (Figure $1 \mathrm{C}$ ). This validates that the simple ADFF analysis technique extracts sufficient information from the Airy Disc patterns to determine the size and aspect ratio of a diffracting object.

There are three measurement errors associated with the Lensless microscope. For objects of size sub $6 \mu \mathrm{m}$ the error is dominated by the diffraction limit error of $0.3 \mu \mathrm{m}$. For objects over this range the error is $5 \%$. This error, associated with ADFF calculations, is attributed to mask calculation error, interaction between close diffracting objects and imaging artefacts. For measurements from the optical microscope we calculate an error of $5 \%$. This error can be attributed to interaction with surface structures and focal plane through the object.

Fission yeast cells arriving on the surface have been growing in liquid culture for $\sim 10$ hours and are in logarithmic growth. The distribution of lengths for cells in logarithmic phase is expected to range from $7-14 \mu \mathrm{m}$ in solution (Mitchison and Nurse 1985). As shown in Figure 3A/B the length distributions for the cells placed on the surface in the flow cell at $t 0$ is between 6.2 and $14.1 \mu \mathrm{m}$, reflecting those previously reported (Pyati et al. 2011). The probability density function histogram of these data can also be used to predict the length of time the cell is in each stage of the cell cycle, with this data set showing that the cell spends more than $50 \%$ of the time at a length of less than $10 \mu \mathrm{m}$.

The distribution of single cell widths is known to be highly controlled and restricted to within a small range (Das et al. 2007) (Kelly and Nurse 2011; Villar-Tajadura et al. 2008). We demonstrate in Figure $3 \mathrm{C}$ and D that our calculated distribution shadows the published distribution, a validation of the technique.

$L_{B}$ is calculated as half the length at cytokinesis. $L_{B}$ has a bimodal distribution. The larger, shorter distribution contains all cells which divided at an expected length, within the range $\sim 13-15 \mu \mathrm{m}$ (Mitchison and Nurse 1985). The smaller distribution of longer cells represents a population with an apparent size larger than expected. This can be explained in three ways: the cells have grown significantly longer than expected; the cells are in a state of rapid growth, entering G1 and S-phase before completing cytokinesis, they will contain two nuclei but grow as one cell (Mitchison and Nurse 1985) or; the cells have undergone cytokinesis but remain touching end on end. If the cells do not 'raft' the Lensless microscope will not distinguish between the two cells according to the Rayleigh limit of resolution (Born and Wolf 1999).

The latter two scenarios have been excluded as in our analysis all cells have been characterised optically and those with a visible septum and/or two nuclei have been removed from the $L_{B}$ distribution (see Fig. A. 1). We can conclude that the cells exhibiting a long length at cytokinesis could be a naturally occurring cell cycle phenotype or have an altered ratio of cell growth and cell division, which would cause a fluctuation in length. 
From the estimate of $L_{B}$ and the calculation, from the growth curves of the data, the time to cytokinesis adjusted for the length of the lag period and the length of the cell at t0mins we can calculate the estimated length of the cell cycle at $25^{\circ} \mathrm{C}$ using this simple equation:

$$
\text { (1): }\left(t_{A}-\lambda\right)=\left(L_{B} / l\right) t_{c e l l}
$$

where $t_{A}$ is the time of cytokinesis in minutes, $\lambda$ is is the length of the lag period in minutes, $L_{B}$ is the birth length in $\mu \mathrm{m}, 1$ is the length of the major axis at $t 0$ in $\mu \mathrm{m}$ and $t_{c e l l}$ is the cell cycle length in minutes.

The distribution of $t_{\text {cell }}$ is displayed in Figure 5.

\section{INSERT FIGURE 5}

The published length of the cell cycle for these experimental conditions converges on 3.1 hours (Martin and Chang 2005), the median of these data converges to $3.5( \pm 0.2)$ hours, is not normally distributed (SW and AD $\mathrm{P}<0.001$ ) and had a skewness coefficient of -0.2828 . The data set slightly over estimates the length of the cell cycle. In this system cells are only said to have undergone cytokinesis if the cells have changed angle relative to one and other. The $t_{c e l l}$ calculation assumes that this change happens immediately post division. This is unlikely to be the case and accounts for the slight over estimation in cell cycle length.

\section{Conclusions}

We have shown that the simple Airy Disc First Fringe analysis calculates a reproducible and reliable determination of the scattering dimensions of the diffracting particles. We are able to accurately measure the size of particles below $6 \mu \mathrm{m}$ to the diffraction limit and the size of particles above $6 \mu \mathrm{m}$ to within $5 \%$. The instrument is stable over time and temperature and we are able to measure the growth parameter distributions from $N=100$ cells. The distributions have median values that are consistent with the literature values in each case but the non-normality of the distributions suggests that there are growth phenotype sub-populations. These outliers in the populations can be used to identify growth phenotypes, such as persister cells, for future study.

\section{References}

2009. Beyond the diffraction limit. Nat Photon 3(7), 361-361.

Anderson, T.W., Darling, D.A., 1952. Asymptotic Theory of Certain "Goodness of Fit" Criteria Bassed on Stochastic Processes. Annals of Mathematical Statistics 23(2), 193.

Bendall, S.C., Nolan, G.P., 2012. From single cells to deep phenotypes in cancer. Nat Biotech 30(7), 639-647.

Bishara, W., Su, T.-W., Coskun, A.F., Ozcan, A., 2010. Lensfree on-chip microscopy over a wide field-of-view using pixel super-resolution. Optical Express 18(11), 11181. 
Born, M., Wolf, E., 1999. Principles of Optics. Cambridge University Press, Cambridge.

Cui, X., Lee, L.M., Heng, X., Zhong, W., Sternberg, P.W., Psaltis, D., Yang, C., 2008. Lensless high-resolution on-chip optofluidic microscopes for Caenorhabditis elegans and cell imaging. Proceedings of the National Academy of Sciences 105(31), 10670-10675.

Das, M., Wiley, D.J., Medina, S., Vincent, H.A., Larrea, M., Oriolo, A., Verde, F., 2007. Regulation of Cell Diameter, For3p Localization, and Cell Symmetry by Fission Yeast Rho-GAP Rga4p. Molecular Biology of the Cell 18(6), 2090.

Fantes, P.A., 1977. Control of cell size and cycle time in Schizosaccharomyces pombe. Journal of Cell Science 24, 51.

Hall-Stoodley, L., Costerton, J.W., Stoodley, P., 2004. Bacterial biofilms: from the Natural environment to infectious diseases. Nat Rev Micro 2(2), 95-108.

Henderson, A.R., 2006. Testing Experimental Data for Univariate Normality. Clinica Chimica Acta 366, 112.

Isikman, S.O., Bishara, W., Mavandadi, S., Yu, F.W., Feny, S., Lau, R., Ozcan, A., 2011. Lens-free optical tomographic microscope with a large imaging volume on a chip. Proceedings of the National Academy of Sciences of the United States of America 108(18), 7296.

Kelly, F.D., Nurse, P., 2011. Spatial control of Cdc42 Activation Determines Cell Width in Fission Yeast. Molecular Biology of the Cell 22, 11.

Lange, D., Storment, C.W., Conley, C.A., Kovac, G.T.A., 2005. A microfluidic shadow imaging system for the study of the nematode Caenorhabditis elegans in space. Sensors and Actuators B: Chemical 107(2), 11.

Lewis, K., 2010. Persister Cells. Annual Review of Microbiology 64, 357.

Martin, S.G., Chang, F., 2005. New End Take Off - Regulating Cell Polarity during the Fission Yest Cell Cycle. Cell Cycle 4(8), 4046.

Matlab, 1994-2013. The MathWorks, Inc. 3 Apple Hill Drive, Natick, Massachusetts 01760 USA. Mathworks.

Mitchison, J.M., Nurse, P., 1985. Growth in the cell length in the fission yeast Schizosaccharomyces pombe Journal of Cell Science 75, 357.

Mitchison, J.M., Walker, P.M.B., 1959. RNA synthesis during the cell life cycle of a fission yeast Schizosaccharomyces pombe. Experimental Cell Research 16(1), 49-58.

Moreno, S., Klar, A., Nurse, P., 1991. Molecular Genetics Analysis of Fission Yeast Schizosaccharomyces pombe. Methods in Enzymology 194, 795. 
Ozcan, A., Demirci, U., 2008. Ultra wide-field lens-free monitoring of cells on-chip. Lab on a Chip 8(1), 98.

Peeling, R.W., Mabey, D., 2010. Point-of-care tests for diagnosing infections in the developing world. Clinical Microbiology and Infection 16(8), 1062.

Pyati, R., Elvir, L.L., Charles, E.C., Seenath, U., Wolkow, T.D., 2011. Imageing Flow Cytometric Analysis of Schizosaccharomyces pombe Morphology. Journal of Yeast and Fungal Research 2(7), 106.

Sauer, K., Camper, A.K., Ehrlich, G.D., Costerton, J.W., Davies, D.G., 2002. Pseudomonas aeruginosa Displays Multiple Phenotypes during Development as a Biofilm. Journal of Bacteriology 184(4), 1140.

Schnars, U., Jueptner, W., 2005. Digital Holography. Digital Holography, pp. 41-69. Springer Berlin Heidelberg.

Seo, S., Isikman, S.O., Sencam, I., Mudanyali, O., Su, T.-W., Bishara, W., Erlinger, A., Ozcan, A., 2010. High-throughput Lens-free Blood analysis on a chip. Analytical Chemistry 82(11), 4621.

Seo, S., Su, T.-W., Erlinger, A., Ozcan, A., 2008. Multi-color LUCAS: Lensfree On-chip Cytometry Using Tunable Monochromatic Illumination and Digital Noise Reduction. Cellular and Molecular Bioengineering 1(2-3), 146.

Shapiro, S.S., Wilk, M.B., 1965. An analysis of variance test for normality (complete samples). Biometrika 52(3-4), 591.

Snell, V., Nurse, P., 1993. Investigations into the Control of Cell Form and Polarity: the use of Morphological Mutants in Fission Yeast. Development. Supplement, 289.

Su, T.-W., Isikman, S.O., Bishara, W., Tseng, D., Erlinger, A., Ozcan, A., 2010. Multiangle lensless digital holography for depth resolved imaging on a chip. Optical Express 18(9), 9690.

Su, T.-W., Seo, S., Erlinger, A., Ozcan, A., 2009. High-Throughput Imaging and Characterization of a Heterogeneous Cell Solution on a Chip. Biotechnology and Bioengineering 102(3), 856.

Tseng, D., Mudanyali, O., Oztoprak, C., Isikman, S.O., Sencam, I., Yaglidere, O., Ozcan, A., 2010. Lensfree microscopy on a cellphone. Lab on a Chip 10, 1787.

Villar-Tajadura, M.A., Coll, P.M., Madrid, M., Cansado, J., Santos, B., Pérez, P., 2008. Rga2 is a Rho2 GAP that regulates morphogenesis and cell integrity in S. pombe. Molecular Microbiology 70(4), 867-881. 
Xu, W., Jericho, M.H., Meinertzhagen, I.A., Kreuser, H.J., 2001. Digital in-line holography for biological applications. Proceedings of the National Academy of Sciences of the United States of America 98(20), 11301.

\section{Figure Captions}

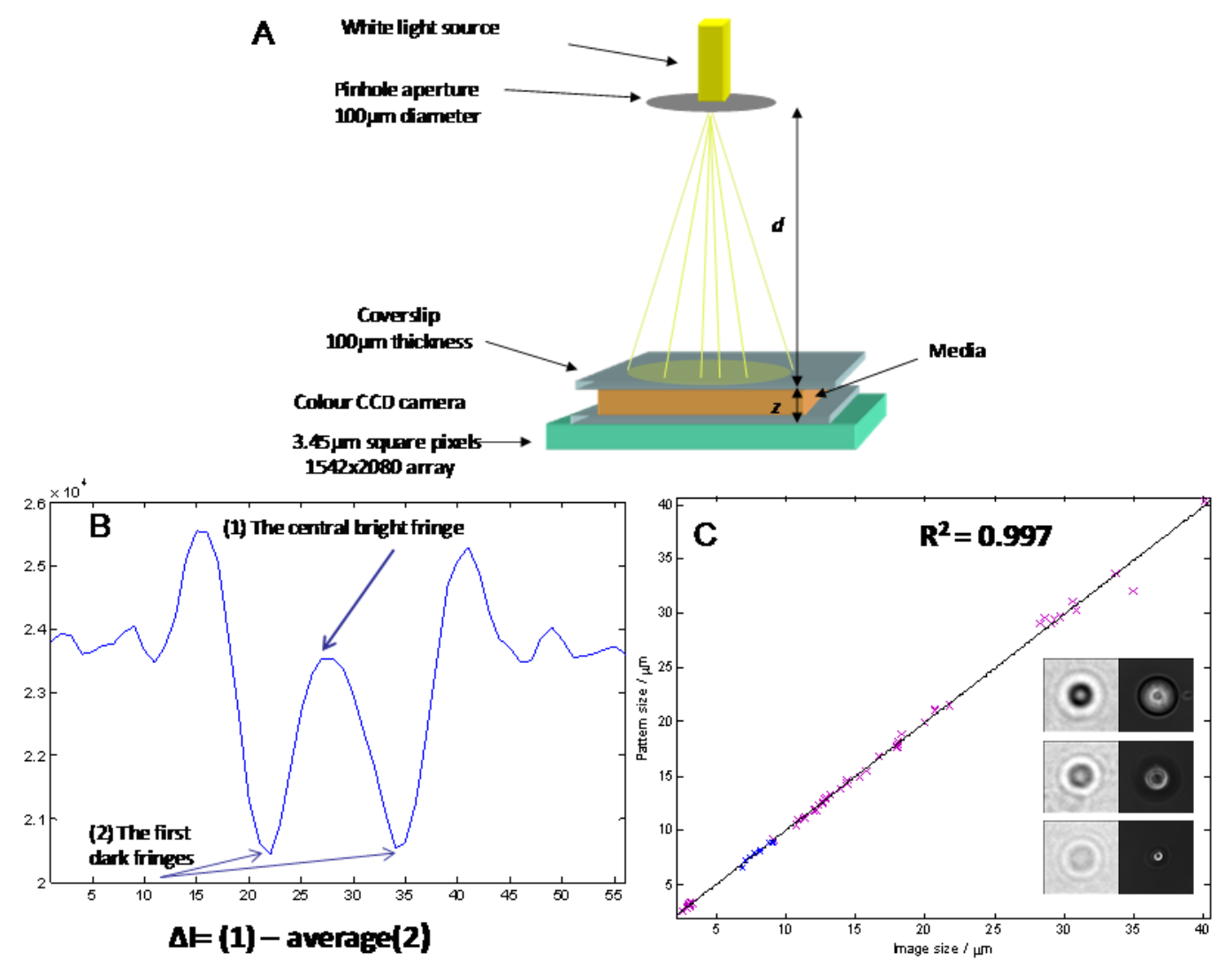

Fig.1. The Lensless microscope configuration: A: a schematic of the microscope; $\mathrm{C}$ a calibration curve comparing the lengths derived from the intensity differences from centre and first fringe of the Airy Disc (B) and measured from the optical image (insert) 


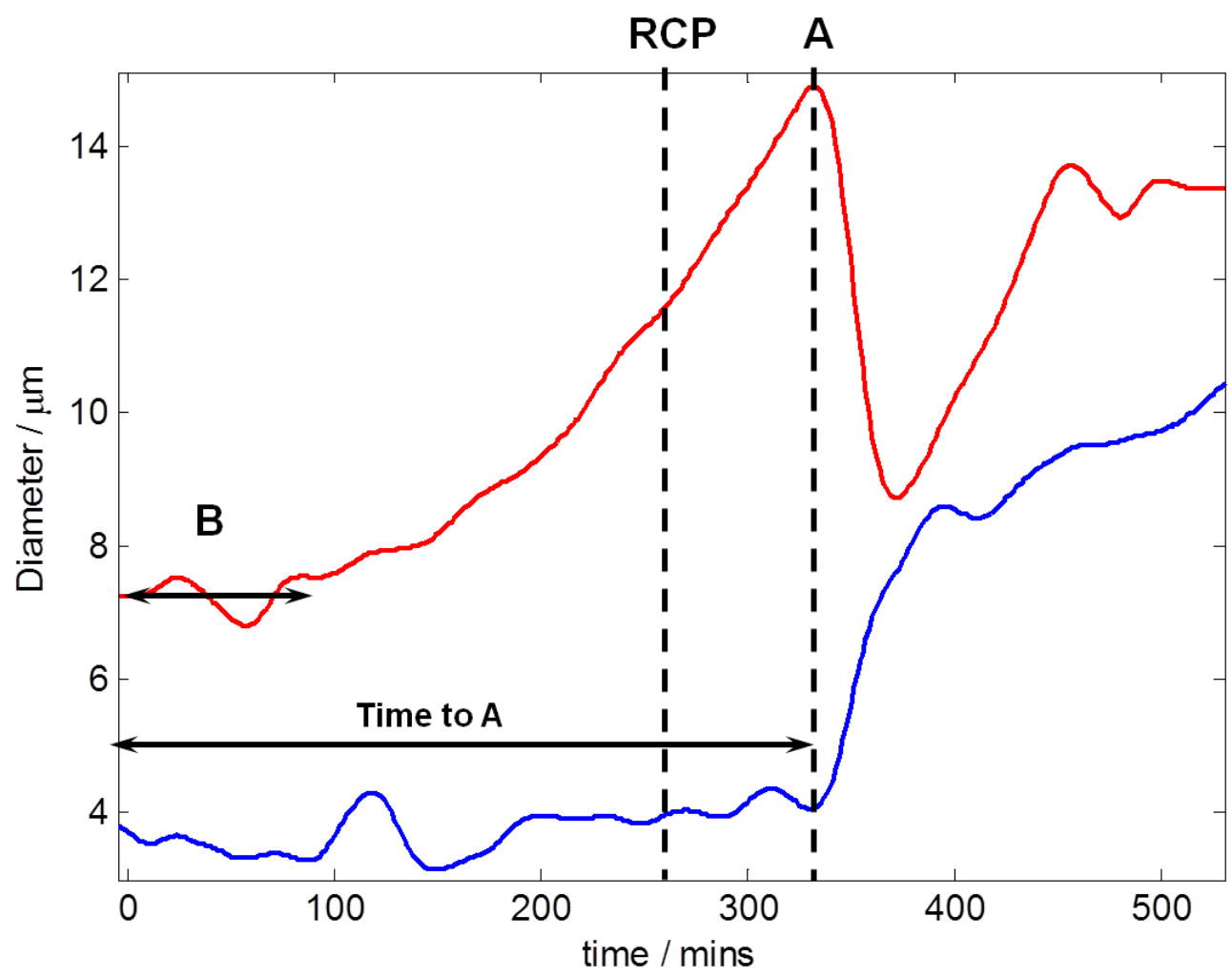

Fig.2. A typical growth curve for a single S.pombe cell from birth to cytokinesis, along both the major (red) and minor (blue) axes. The parameters of lag period $(\lambda)$, time to cytokinesis (A) and the RCP are marked. 

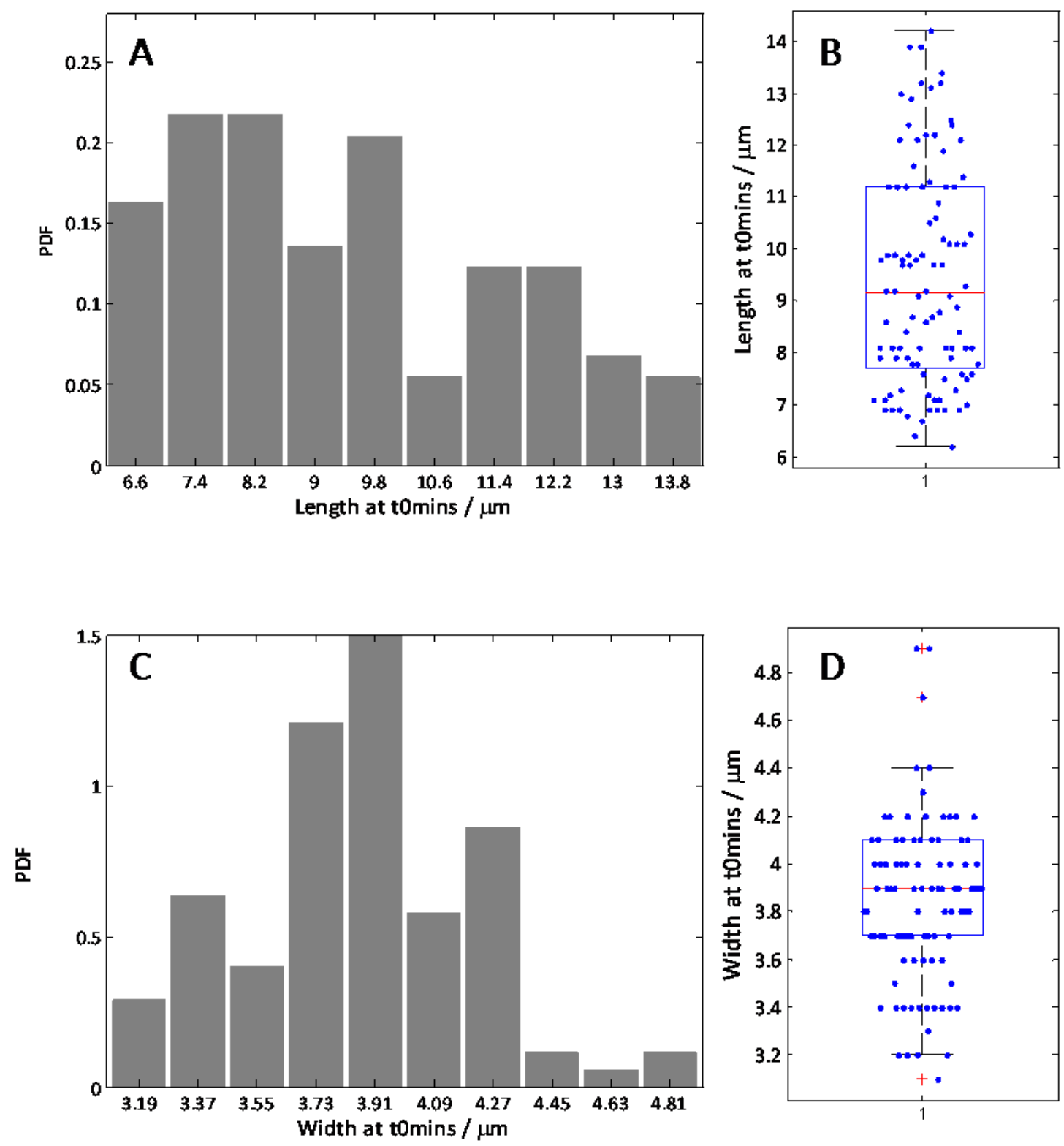

Fig.3 A, Normalised Histogram for the distribution of the major axis length of $N=100$ S.pombe cells at $t=0$ mins; B, the Box plot for this distribution, showing the median (red) and the 25th and 75th percentiles. The length distribution have a median of $9.2(+0.6-$ $0.9) \mu \mathrm{m}$ and is not normal distributed (P value $<0.001$ ); C, Normalised Histogram for the distribution of the minor axis width of $N=100$ S.pombe cells at $t=0$ mins; $\mathrm{D}$, the Box plot for the distribution. The width distribution has a median of $3.9( \pm 0.1) \mu \mathrm{m}$ and is not normal distributed $(\mathrm{P}$ value $<0.001)$. 

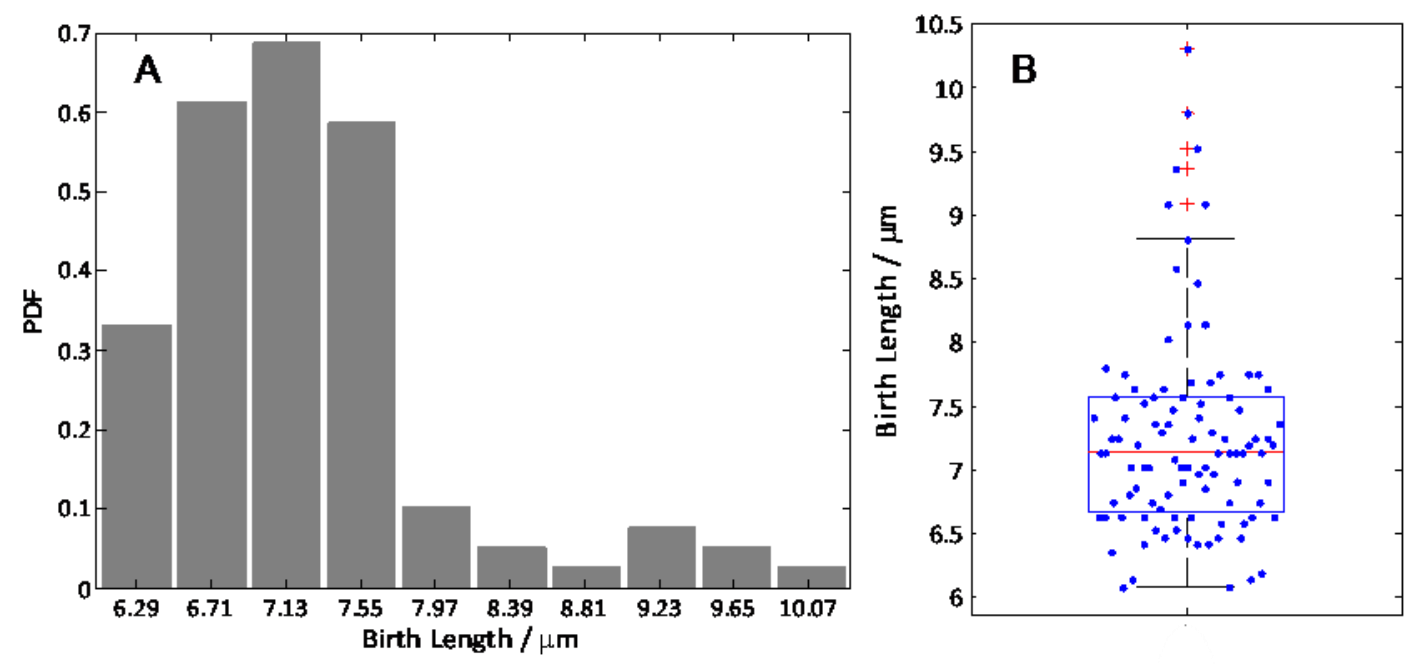

Fig.4. A, Normalised Histogram for the distribution of $L_{B}$ of $N=100$ S.pombe cells; B, the Box plot for this distribution. The distribution converges on a median of $7.3( \pm 0.2) \mu \mathrm{m}$ and is not normal ( $\mathrm{P}$ values $<0.001$ ). $9 \%$ of the cells have a calculated $L_{B}$ in the second, high distribution, these are the cells which have undergone cytokinesis at a length significantly longer than predicted. The distribution of birth length of the cells has a median of $7.3( \pm 0.2) \mu \mathrm{m}$, a skewness coefficient of 1.49 and is not normally distributed (SW test, $\mathrm{P}<0.001)$.
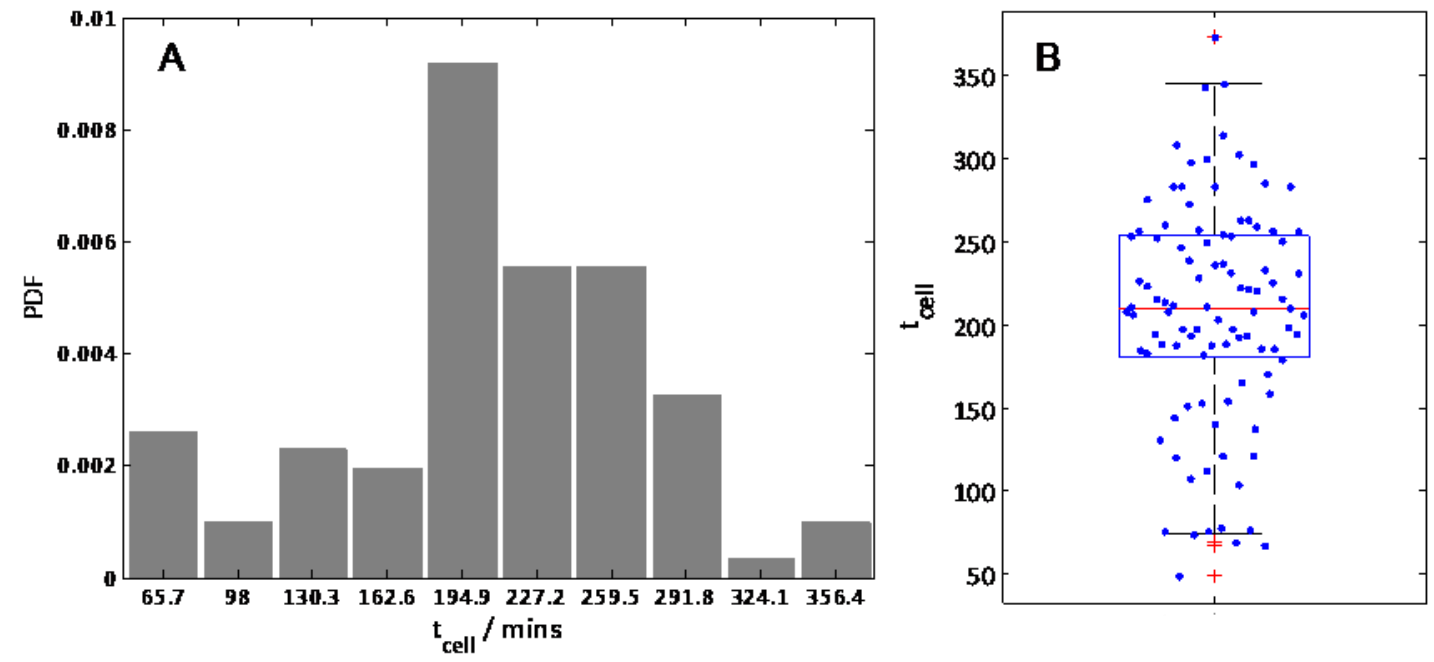

Fig.5. A, Normalised Histogram for the distribution of $t_{\text {cell }}$ for $N=100$ S.pombe cells; D, the Box plot for this distribution. The distribution converges on a median of $208( \pm 12)$ mins and is not normal ( $\mathrm{P}$ value $<0.001)$ 

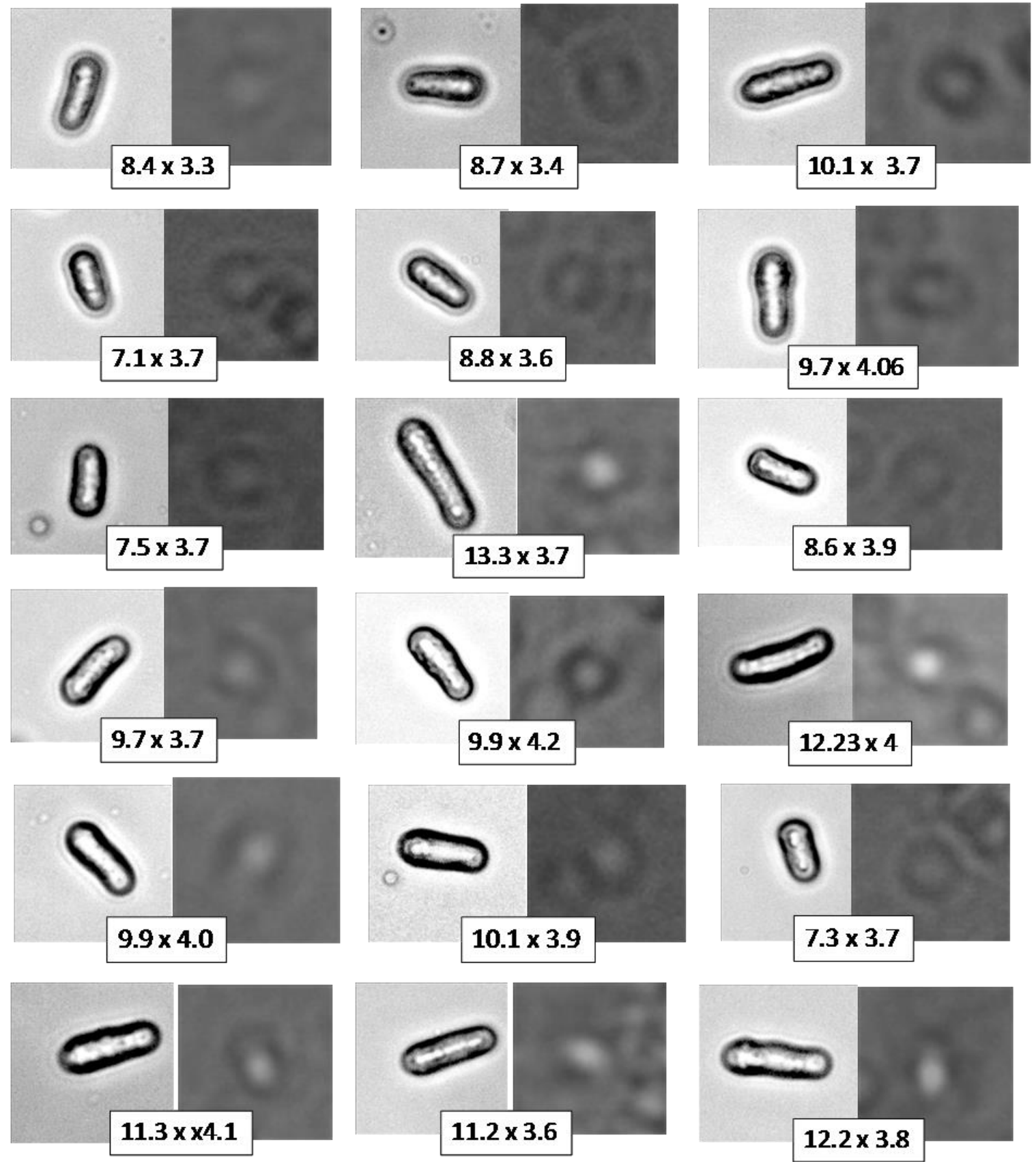

Fig. A. 1. Airy Patterns for 18 fission yeast cells and the corresponding light microscope image. 